\title{
Embedded graphs whose links have the largest possible number of components
}

\author{
Stephen Huggett \\ School of Computing and Mathematics, University of Plymouth, Plymouth PLA 8AA, UK \\ Israa Tawfik * \\ Department of Mathematics, College of Education for Girls, Tikrit University, Tikrit, Iraq
}

Received 27 May 2013, accepted 10 March 2015, published online 11 March 2015

\begin{abstract}
We derive the basic properties of graphs embedded on surfaces of positive genus whose corresponding link diagrams have the largest possible number of components.
\end{abstract}

Keywords: Embedded graphs, medial, components of links.

Math. Subj. Class.: 05C10, 57M25

\section{Introduction}

A graph $G$ embedded in a surface determines a link diagram $D(G)$, which has a certain number $\mu$ of components.

The relationship between the graph and the link diagram is through the crossing which replaces each edge of the graph, shown in figure 1. In this article, we are only interested in $\mu$. So at each crossing, the choice of the over-crossing strand does not matter, and we are therefore actually considering a link universe rather than a diagram of a particular link. However, we will for simplicity refer to a link diagram.

The relationship between a graph and the number of components in the corresponding link diagram has been studied by several people. It is shown in [6] that

$$
T(G ;-1,-1)=(-1)^{q(G)}(-2)^{\mu(D(G))-1},
$$

where $T(G ; x, y)$ is the Tutte polynomial and $q(G)$ is the number of edges in $G$. In [4], equation (1.1) is generalised to the projective plane and the torus, while in [7] $T(G ;-1,-1)$

\footnotetext{
* Supported by the Ministry of Higher Education and Scientific Research, Iraq.

E-mail addresses: s.huggett@plymouth.ac.uk (Stephen Huggett), israa.tawfik@plymouth.ac.uk (Israa Tawfik)
} 


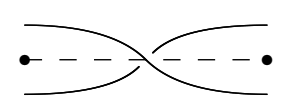

Figure 1: A crossing replacing an edge. The curved lines are strands of the link diagram, and the dashed straight line joining the two vertices is the edge of the graph replaced by the crossing.

is calculated for fans, wheels, and 2-sums of graphs. The number $\mu$ is the same as the number of "straight-ahead" walks in medial graphs, as described in [8]. The focus in [3] is to characterize the plane graphs $G$ whose $\mu(D(G))$ is as large as possible, which is the cycle rank plus one; these are the "extremal" graphs. Maximising $\mu$ is also our principal interest here, although we will study graphs embedded on various orientable surfaces.

In section 2 we show how $\mu$ depends on the blocks of the graph, we note that $\mu$ does not change when the graph undergoes a "graph Reidemeister move" or an "embedded" Y $\leftrightarrow \Delta$ move, and we show that $\mu$ cannot change very much when an edge is added to the graph. In section 3 we study plane graphs. Many of our results replicate those in [3], although our emphasis is different because we are preparing to work on other surfaces.

Let $g$ be the genus of a surface. Then section 4 shows how to extend many of the results of section 3 to graphs embedded on surfaces with $g>0$. In particular, in Theorem 4.3 we show that $\mu \leq f+2 g$, where $f$ is the number of faces in the embedding, so that the extremal graphs now have $\mu=f+2 g$. In Theorem 4.5 we give a list of some graph operations which preserve the extremal property, and in Corollary 4.6 and Theorem 4.7 we give some local consequences of this property.

We finish in section 5 with some observations on other possible values of $\mu$. For plane graphs, the case when $\mu$ is equal to the cycle rank is considered in [5], where it is found that this class of graphs is quite severely constrained. We comment on two other cases: the case when $\mu=1$, and the Petersen and Heawood families.

It is a pleasure to thank Iain Moffatt for many interesting discussions.

\section{Basic results}

Our first theorem comes from the connected sum operation on links.

Theorem 2.1. Let $G$ be a graph with blocks $B_{1}, B_{2}, \ldots, B_{k}$. Then

$$
\mu(D(G))=\sum_{i=1}^{k} \mu\left(D\left(B_{i}\right)\right)-(k-1) .
$$

Proof. For any two adjacent blocks $B_{i}$ and $B_{i+1}$ of $G$ with common vertex $v$, the two strands at $v$ must be part of the same component. So splitting $G$ at $v$ into two graphs increases the number of components by one. See figure 2 .

Therefore, splitting $G$ into its $k$ blocks increases the number of components by $k-1$, and hence the result.

Theorem 2.2. Let $G$ be a graph with a bridge e. Then

$$
\mu(D(G))=\mu(D(G / e))
$$




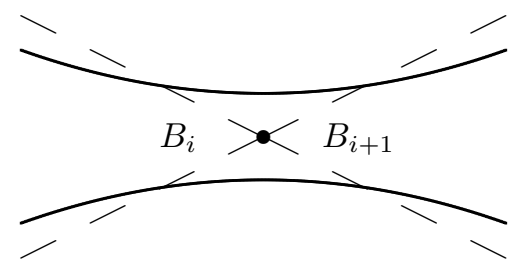

Figure 2: The curved lines are two strands of a single component at a cut vertex of the graph, which separates blocks $B_{i}$ and $B_{i+1}$. The dashed straight lines are edges of the graph.

Proof. Let $G_{1}$ and $G_{2}$ be the two components of $G \backslash\{e\}$, and let $B$ be the block in $G$ containing $e$. Then by Theorem 2.1

$$
\begin{aligned}
\mu(D(G)) & =\mu\left(D\left(G_{1}\right)\right)+\mu\left(D\left(G_{2}\right)\right)+\mu(D(B))-2 \\
& =\mu\left(D\left(G_{1}\right)\right)+\mu\left(D\left(G_{2}\right)\right)-1,
\end{aligned}
$$

because $\mu(D(B))=1$. However, $G /\{e\}$ consists of blocks $G_{1}$ and $G_{2}$, so by Theorem 2.1 again

$$
\mu(D(G /\{e\}))=\mu\left(D\left(G_{1}\right)\right)+\mu\left(D\left(G_{2}\right)\right)-1,
$$

and hence the result.

Theorem 2.3. Let $G$ be a graph with parallel edges $e_{1}$ and $e_{2}$ bounding a disc. Then

$$
\mu(D(G))=\mu\left(D\left(G \backslash\left\{e_{1}, e_{2}\right\}\right)\right) .
$$

If, instead, $e_{1}$ and $e_{2}$ are not parallel edges, but are incident with a common vertex of degree 2 , then

$$
\mu(D(G))=\mu\left(D\left(G /\left\{e_{1}, e_{2}\right\}\right)\right) .
$$

Proof. This follows immediately from the Reidemeister 2 move (see figure 3) on the link diagrams, which evidently preserves $\mu$.
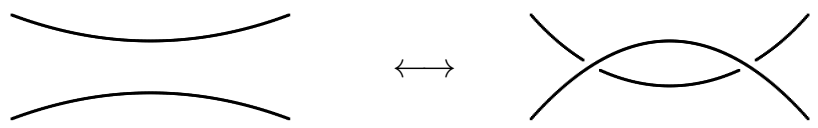

Figure 3: The Reidemeister 2 move on a link diagram.

We next consider the $\mathrm{Y} \leftrightarrow \Delta$ moves. These replace a "Y" by a triangle, or vice versa, as in figure 4. For our purposes, we need the graph to be embedded in a surface and the triangle to bound a disc on that surface. Then we refer to the $\mathrm{Y} \leftrightarrow \Delta$ move as embedded. Otherwise it is an abstract $\mathrm{Y} \leftrightarrow \Delta$ move.

Theorem 2.4. If $G_{1}$ and $G_{2}$ are related by embedded $Y \leftrightarrow \Delta$ moves, then

$$
\mu\left(D\left(G_{1}\right)\right)=\mu\left(D\left(G_{2}\right)\right) .
$$



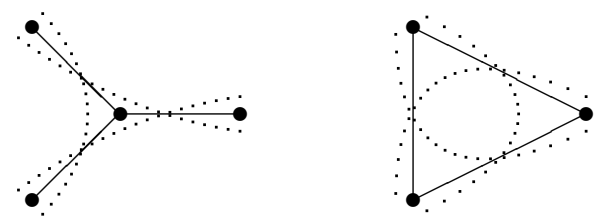

Figure 4: The effect of a $\mathrm{Y} \leftrightarrow \Delta$ move. In this figure the curved dotted lines are the strands of the link diagram, while the straight lines are the edges of the graph.

Proof. This is evident from figure 4.

Finally in this section we note that adding an edge cannot change the number of components very much.

Theorem 2.5. Let e be a new edge connecting two vertices in the same face of a graph $G$, this face being a disc. Then

$$
\mu(D(G))-1 \leq \mu(D(G+e)) \leq \mu(D(G))+1 .
$$

Proof. If $e$ is a loop bounding a disc then

$$
\mu(D(G))=\mu(D(G+e))
$$

so the result holds. If $e$ is a loop not bounding a disc, or $e$ is not a loop, then there are two cases: we refer to figure 5, where the face is labelled $\mathrm{F}$.
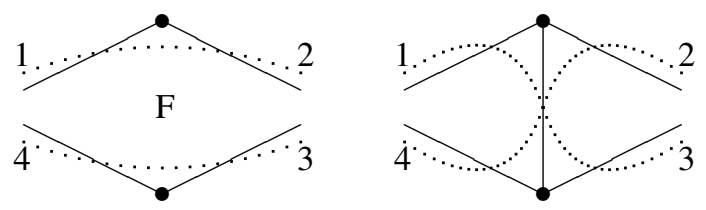

Figure 5: The effect of adding an edge in a face F. The $\operatorname{arcs} \alpha$ (joining 1 and 2) and $\beta$ (joining 3 and 4) may or may not be in different components. (Again, the curved dotted lines are the strands of the link diagram, while the straight lines are the edges of the graph.)

(1) If the arcs $\alpha$ (joining 1 and 2) and $\beta$ (joining 3 and 4) are contained in different components of $D(G)$, then

$$
\mu(D(G+e))=\mu(D(G))-1
$$

(2) If the arcs $\alpha$ and $\beta$ are contained in the same component of $D(G)$, then there are two further cases.

(a) Along this one component, if the order of the four endpoints of the two $\operatorname{arcs} \alpha$ and $\beta$ is $1,2,3,4$ then

$$
\mu(D(G+e))=\mu(D(G))
$$


(b) If the order of the four endpoints of the two $\operatorname{arcs} \alpha$ and $\beta$ is $1,2,4,3$ then

$$
\mu(D(G+e))=\mu(D(G))+1 .
$$

\section{Extremal plane graphs}

Theorem 3.1. Let $G$ be a connected plane graph. Then

$$
1 \leq \mu(D(G)) \leq f(G)
$$

Proof. Let $T$ be a spanning tree of the graph $G$. Then $f(T)=1$ and $\mu(D(T))=1$, so the theorem is true for $T$.

Now add, one by one, edges to $T$ in order to obtain $G$. The intermediate graphs are

$$
G_{1}, G_{2}, \ldots, G_{s-1}
$$

We obtain a sequence of graphs

$$
T=G_{0}, G_{1}, \ldots, G_{s-1}, G_{s}=G
$$

The insertion of an edge increases the number of faces by exactly one, so for $i=0, \ldots, s-$ 1 we have

$$
f\left(G_{i+1}\right)=f\left(G_{i}\right)+1=f\left(G_{0}\right)+i+1 .
$$

By Theorem 2.5

$$
\begin{aligned}
\mu\left(D\left(G_{i+1}\right)\right) & \leq \mu\left(D\left(G_{i}\right)\right)+1 \\
& \leq \mu\left(D\left(G_{0}\right)\right)+i+1 .
\end{aligned}
$$

Since $\mu\left(D\left(G_{0}\right)\right)=f\left(G_{0}\right)$, we must have $\mu\left(D\left(G_{i+1}\right)\right) \leq f\left(G_{i+1}\right)$ for each $i$, which means that $\mu(D(G)) \leq f(G)$.

If $G$ is a connected plane graph then $G$ is called extremal if

$$
\mu(D(G))=f(G) .
$$

A face of a plane graph is called even if it has an even number of edges.

Theorem 3.2. If $G$ is extremal then each face of $G$ is even.

Proof. Let $T$ be a spanning tree of $G$, and let

$$
T=G_{0}, G_{1}, G_{2}, \ldots, G_{s}=G
$$

be the sequence of graphs in the proof of Theorem 3.1. Since $T$ and $G$ are extremal then each $G_{i}$ in the sequence is extremal. Otherwise $\mu\left(D\left(G_{i}\right)\right)<f\left(G_{i}\right)$, and then from Theorem 2.5 we would have $\mu\left(D\left(G_{i+1}\right)\right)<f\left(G_{i+1}\right)$ and eventually $\mu(D(G))<f(G)$. 
$T$ has one even face. Suppose that there is a graph in this sequence with an odd face, and let $G_{i+1}$ be the first such graph. $G_{i+1}=G_{i} \cup e$ where $e$ has been inserted into a necessarily even face in $G_{i}$, creating two odd faces $f_{1}$ and $f_{2}$ in $G_{i+1}$.

Because all the graphs in the sequence are extremal, we must be in case $\mathbf{2 b}$ of Theorem 2.5. Choose the component of $D\left(G_{i+1}\right)$ which includes the arc 13 . This component contains exactly one of the faces $f_{1}$ or $f_{2}$, suppose it is $f_{1}$, and the component defines an even circuit in the edges of $G_{i+1}$. But the faces inside this circuit are all even except $f_{1}$, because all except $f_{1}$ come from $G_{i}$, which is a contradiction.

Corollary 3.3. If $G$ is extremal then $G^{*}$ is eulerian.

The converse of this corollary is not true. For example, let $G$ be the dual graph of $K_{2,2}$. Then $G^{*}$ is eulerian but $G$ is not extremal.

Corollary 3.4. If $G$ is extremal then $G$ is bipartite.

Define $\delta(G)$ to be the minimum degree of $G$.

Theorem 3.5. If $G$ is extremal, then $\delta(G)<3$.

Proof. Let

$$
T=G_{0}, \ldots, G_{i}, G_{i+1}, \ldots, G_{s}=G
$$

be the sequence of extremal graphs in Theorem 3.2, in which

$$
G_{0}, \ldots, G_{i}
$$

all have $\delta(G)<3$, while

$$
G_{i+1}, \ldots, G_{s}
$$

all have $\delta(G) \geq 3$. Then $\delta\left(G_{i}\right)$ must be 2 .

When we add an edge to $G_{i}$ to get $G_{i+1}$ we contradict Theorem 3.1, because in $G_{i+1}$ the number of faces has increased but the number of components has not: see figure 6 . So $i=s$.
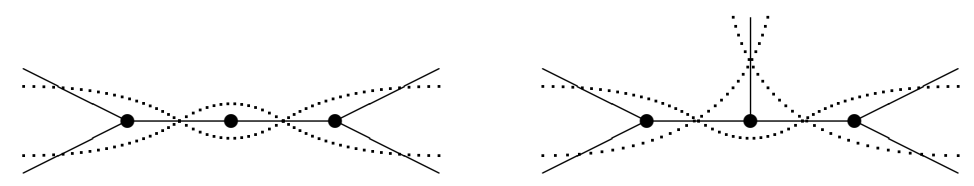

Figure 6: Adding an edge to $G_{i}$ to obtain $G_{i+1}$.

Lemma 3.6. If $G$ is extremal then it has no loops, and any parallel edges must be between cut-vertices. 
Proof. $G$ cannot have a pair $e_{1}, e_{2}$ of parallel edges between vertices which are not cutvertices, because if it did then

$$
\begin{aligned}
f(G) & =\mu(D(G)) \\
& =\mu\left(D\left(G \backslash\left\{e_{1}, e_{2}\right\}\right)\right) \quad \text { by Theorem } 3 \\
& \leq f\left(G \backslash\left\{e_{1}, e_{2}\right\}\right) \quad \text { by Theorem } 3.1 \\
& \leq f(G)-2 .
\end{aligned}
$$

(This argument fails if the parallel edges are between cut-vertices because Theorem 3.1 needs a connected graph.) Similarly, $G$ cannot have a loop $e$, because if it did then

$$
f(G)=\mu(D(G))=\mu(D(G \backslash\{e\})) \leq f(G \backslash\{e\})=f(G)-1 .
$$

Lemma 3.7. Let $G$ be connected, simple, and non-trivial. Then $G^{*}$ is not extremal.

Proof. If $G$ is connected, simple, and non-trivial then $G^{*}$ has no vertices of degree 1 or 2 . So from Theorem $3.5 G^{*}$ is not extremal.

Lemma 3.8. Let $G$ be connected, simple, and non-trivial. Then

$$
\mu(D(G))<p .
$$

Proof. From Lemma $3.8 G^{*}$ is not extremal. Therefore

$$
\mu\left(D\left(G^{*}\right)\right)<f\left(G^{*}\right)
$$

and hence

$$
\mu(D(G))<p(G)
$$

as required.

Theorem 3.9. Let $G$ be a simple connected plane graph with a non-trivial dual. Then $G$ is extremal if and only if there is an even number of edges between each pair of vertices of $G^{*}$.

Proof. Suppose there were a pair of vertices of $G^{*}$ with an odd number of edges joining them. Delete all loops and all pairs of parallel edges in $G^{*}$, to obtain a simple graph $H$ (not necessarily connected) with at least one edge, and such that $\mu\left(D\left(G^{*}\right)\right)=\mu(D(H)$. Each component of $H$ either has $\mu=1$ or $\mu$ less than the number of vertices in that component, by Lemma 3.8. Therefore

$$
\mu(D(G))=\mu\left(D\left(G^{*}\right)\right)=\mu\left(D(H)<p(H)=p\left(G^{*}\right)=f(G),\right.
$$

contradicting that $G$ is extremal.

If $G^{*}$ has an even number of edges between each pair of vertices, then we delete all pairs of parallel edges in $G^{*}$, to obtain a graph with no edges. By Theorem 3 this does not change the number of components, and so

$$
\mu(D(G))=\mu\left(D\left(G^{*}\right)\right)=v\left(G^{*}\right)=f(G) .
$$


Theorem 3.10. Let $G$ be a connected plane graph. Then the following statements are true.

(a) Let e be a bridge of $G$. Then $G / e$ is extremal if and only if $G$ is extremal.

(b) Let $v$ be a vertex of degree 2 with exactly one adjacent vertex. Then $G \backslash v$ is extremal if and only if $G$ is extremal.

(c) Let $v$ be a vertex of degree 2 with two different adjacent vertices $x$ and $y$. Then $G /\{v, x\} /\{v, y\}$ is extremal if and only if $G$ is extremal.

(d) $G$ is extremal if and only if each block of $G$ is extremal.

(e) Let $G$ be extremal and e not a bridge in $G$. Then $G \backslash e$ is extremal.

Proof. (a) This follows from Theorem 2.2.

(b) This follows from Theorem 3 .

(c) This follows from Theorem 3 .

(d) Let $B_{1}, B_{2}, \ldots, B_{k}$ be the blocks of $G$, and suppose that $G$ is extremal. Then from Theorem 2.1 we have

$$
\begin{aligned}
\sum_{i=1}^{k} \mu\left(D\left(B_{i}\right)\right)-k+1 & =\mu(D(G)) \\
& =f(G) \\
& =\sum_{i=1}^{k} f\left(B_{i}\right)-k+1 .
\end{aligned}
$$

Therefore

$$
\sum_{i=1}^{k} \mu\left(D\left(B_{i}\right)\right)=\sum_{i=1}^{k} f\left(B_{i}\right),
$$

and so each $B_{i}$ is extremal. The converse is proved similarly.

(e) Since $e$ is not a bridge, $G \backslash e$ is a connected plane graph and

$$
f(G)=f(G \backslash e)+1 .
$$

By Theorem 2.5

$$
\mu(D(G)) \leq \mu(D(G \backslash e))+1,
$$

but

$$
\mu(D(G))=f(G)=f(G \backslash e)+1,
$$

and so

$$
\mu(D(G \backslash e)) \geq f(G \backslash e) .
$$

Hence by Theorem $3.1 G \backslash e$ is extremal.

Lemma 3.11. Let $G$ be extremal. Then each component of $D(G)$ only ever crosses itself on a bridge. 
Proof. Suppose a component of $D(G)$ crosses itself on the edge $e$ in $G$, not a bridge. By Theorem 3.10, $G \backslash e$ is extremal.

When we delete $e$ the number of components stays the same but the number of faces drops. This is impossible because $G \backslash e$ is extremal.

Note that in the following theorem the graph $G_{i} /\left\{x_{i}, y_{i}\right\}$ is obtained from $G_{i}$ by identifying the vertices $x_{i}$ and $y_{i}$.

Theorem 3.12. Let $G$ be a plane graph. $G$ is extremal if and only if it satisfies one of the following conditions.

(1) $G=K_{1}$

(2) G has a bridge e such that $G \backslash$ e consists of two extremal graphs.

(3) $G$ has edges $e_{i}=x_{i} y_{i}$ for $i=1,2$ such that $G \backslash\left\{e_{1}, e_{2}\right\}$ consists of two disjoint graphs $G_{1}$ and $G_{2}$ with $x_{i}, y_{i} \in V\left(G_{i}\right)$ and $G_{i} /\left\{x_{i}, y_{i}\right\}$ extremal.

Proof. Denote by $f, f_{1}$, and $f_{2}$ the numbers of faces of $G, G_{1}$, and $G_{2}$ respectively. Similarly, denote by $\mu, \mu_{1}$, and $\mu_{2}$ the numbers of components in their link diagrams. Let $G$ be an extremal graph, so that $\mu=f$, and suppose that $G$ has at least one edge.

If $G$ has a bridge $e$, with $G \backslash e=G_{1} \cup G_{2}$, then

$$
f=f_{1}+f_{2}-1 \text {. }
$$

because $G_{1}$ and $G_{2}$ share a common face. Now by Theorem 2.1

$$
\mu=\mu_{1}+\mu_{2}-1
$$

Since $G$ is extremal

$$
\mu_{1}+\mu_{2}-1=\mu=f=f_{1}+f_{2}-1
$$

Therefore

$$
\mu_{1}+\mu_{2}-1=f_{1}+f_{2}-1
$$

which means that

$$
\mu_{1}+\mu_{2}=f_{1}+f_{2}
$$

Since $\mu_{i} \leq f_{i}$ for each $i$, we must now have

$$
\mu_{1}=f_{1}
$$

and

$$
\mu_{2}=f_{2}
$$

as required.

Next, let $G$ be an extremal graph without a bridge. By Theorem 3.5 it must have a vertex $v$ with degree less than 3. However, if $d(v)=0$ then $G=K_{1}$, and if $d(v)=1$ we have a bridge. So $d(v)=2$. Now there are two cases.

(a) If $v$ is adjacent to two distinct vertices $x_{2}$ and $y_{2}$, as in figure 7, then $G_{1}=K_{1}$ (the vertex $v$ ) and $x_{2} \neq y_{2}$ in $G_{2}$. Clearly $\mu_{1}=f_{1}$. Suppose $\mu_{2}$ is the number of components of the link diagram of $G_{2} /\left\{x_{2}, y_{2}\right\}$, and $f_{2}$ is the number of faces of $G_{2} /\left\{x_{2}, y_{2}\right\}$. Then $\mu_{2}=\mu$ and $f_{2}=f$ because the identification of $x$ and $y$ does not affect the number of components of the link diagram of $G_{2}$ or the number of faces of $G_{2}$, which means that $G_{2} /\left\{x_{2}, y_{2}\right\}$ is extremal. 


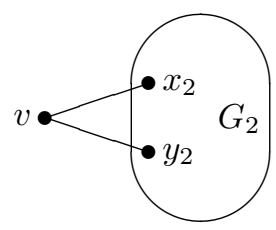

Figure 7: The case $G_{1}=K_{1}$, adjacent to two distinct vertices in $G_{2}$.

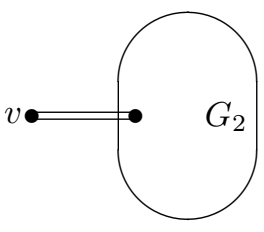

Figure 8: The case $G_{1}=K_{1}$, adjacent twice to a vertex in $G_{2}$.

(b) If $v$ is a vertex adjacent twice to another vertex, as in figure 8, then $G_{1}=K_{1}$ as before, and since $x_{2}=y_{2}$ then $\mu=\mu_{2}+1$ and $f=f_{2}+1$. Since $G$ is extremal then $G_{2} /\left\{x_{2}, y_{2}\right\}$ is extremal.

Conversely, suppose that one of the three conditions holds. Then we will show that $G$ is extremal.

(1) If $G=K_{1}$ then $G$ is extremal because $\mu(G)=f(G)=1$.

(2) If $G$ consists of the two extremal graphs $G_{1}, G_{2}$ and the bridge $e$ between them, then $\mu_{1}=f_{1}$ and $\mu_{2}=f_{2}$ and since $e$ is a bridge then $\mu=\mu_{1}+\mu_{2}-1$. There is a common face between $G_{1}$ and $G_{2}$, so $f=f_{1}+f_{2}-1$, which gives $f=f_{1}+f_{2}-1=\mu_{1}+\mu_{2}-1=\mu$. Therefore $G$ is extremal.

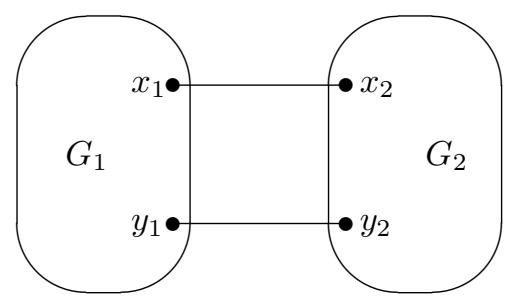

Figure 9: The construction of $G$ from $G_{1}$ and $G_{2}$.

(3) Suppose that the plane graph $G$ is constructed from two connected plane graphs $G_{1}$ and $G_{2}$ by adding two new edges $e_{1}$ and $e_{2}$, where $e_{1}=\left(x_{1}, x_{2}\right), e_{2}=\left(y_{1}, y_{2}\right)$ and $x_{i}, y_{i} \in G_{i}$, as in figure 9. Let $\mu_{i}$ be the number of components of the link diagram of $G_{i} /\left\{x_{i}, y_{i}\right\}$ and $f_{i}$ the number of faces of $G_{i} /\left\{x_{i}, y_{i}\right\}$. Then

$$
f=f_{1}+f_{2}-2
$$


because we will get two new faces, one in $f_{1}$ and another one in $f_{2}$, when we identify $x_{i}$ and $y_{i}$. In order to count the components in the various link diagrams, start with $G_{i} /\left\{x_{i}, y_{i}\right\}$ and then "split" the vertices into $x_{i}$ and $y_{i}$, obtaining the arrangement shown in figure 10 .

Hence

$$
\mu=\mu_{1}+\mu_{2}-2
$$

From equations (3.3) and (3.4), $\mu=f$.

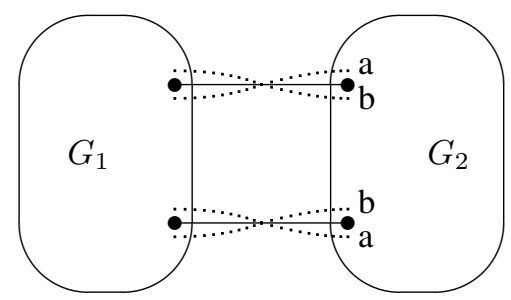

Figure 10: The two components, a and b, crossing from $G_{1}$ to $G_{2}$.

We finish this section by describing ways of constructing new extremal graphs using the operations of 2-sum and tensor product. (These are natural operations on graphs, but are perhaps most easily defined on matroids: see [2].)

Let $G$ and $H$ be any graphs, with distinguished edges $e$ and $f$. The $\mathbf{2}$-sum $G \oplus_{2} H$ along $e$ and $f$ is obtained by identifying the edges $e$ and $f$ to form a new edge, which is then deleted.

The tensor product $G \otimes H$ is obtained by taking the 2-sum of $G$ with $H$ along each edge of $G$ and the edge $f$ in $H$.

For example, when $H$ is the triangle graph, constructing $G \otimes H$ amounts to putting a new vertex of degree two in each edge of $G$. In this case, the embedding of $G \otimes H$ is induced from that of $G$, but this only happens because $H$ is so symmetrical. In general, there may be more than one embedding of $G \otimes H$ for any given embedding of $G$.

Theorem 3.13. Let $G$ be any connected plane graph and $H$ be an odd cycle. Then $G \otimes H$ is extremal.

Proof. This follows from the Reidemeister 2 move on the link diagrams.

Theorem 3.14. Let $G$ be a tree and $H$ be extremal. Then the tensor product $G \otimes H$, in which the distinguished edge in $H$ is not a bridge, is extremal.

Proof. This follows from Theorem 3.10, part (d).

\section{Extremal graphs on surfaces of genus $g$}

Here we will often restrict to cellular embeddings, in which the interior of each face of the embedded graph is homeomorphic to an open disc. (For plane graphs this implies connectedness, of course.)

Given an embedded graph $G$, a spanning subgraph $\psi$ which is connected, has just one face, and is cellularly embedded, is called a pseudo-tree of $G$. A pseudo-tree can be obtained from any cellularly embedded graph by iteratively deleting edges that lie on two faces, until no such edge can be found. 
Firstly, let $G$ be cellularly embedded on the torus. Then each block of $G$ is a connected plane graph except for one, which must be cellularly embedded on the torus.

Theorem 4.1. If $\psi$ is a pseudo-tree cellularly embedded on the torus, then $\mu(D(\psi)) \leq 3$.

Proof. We reduce $\psi$ as follows.

- Contract all bridges in $\psi$. This leaves $\mu(D(\psi))$ unchanged, by Theorem 2.2.

- For each vertex of degree two in $\psi$ whose edges go to distinct vertices, contract both these edges. By Theorem 3 this also leaves $\mu(D(\psi))$ unchanged.

$\psi$ has one meridian $M$ and one longitude $L . M \cap L$ must be non-empty, and if it had more than one connected component then $\psi$ would have more than one face. Now, up to extra meridians or longitudes, there are only four possibilities for the reduced $\psi$, shown in figure 11, and by inspection the link diagrams for $A, B, C$, and $D$ have $\mu=2,1,1$, and 3 respectively. $E$ is the same as $A$, with an extra meridian.

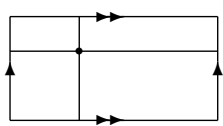

A

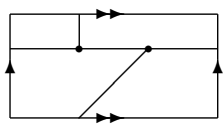

D

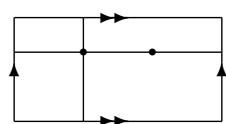

B

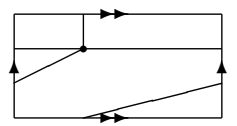

E

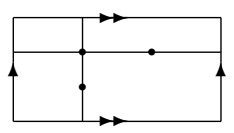

C

Figure 11: $A, B, C$, and $D$ are the four possibilities for the reduced $\psi . E$ is the same as $A$, with an extra meridian.

Theorem 4.2. Let $\psi$ be a pseudo-tree embedded on a surface of genus $g$. Then

$$
\mu(D(\psi)) \leq 1+2 g
$$

Proof. The result is clearly true when $g=0$.

Now suppose that for any pseudo-tree $\psi_{g}$ embedded on a surface $S_{g}$ of genus $g$, we have

$$
\mu\left(D\left(\psi_{g}\right)\right) \leq 1+2 g .
$$

Let $\psi_{g+1}$ be a pseudo-tree embedded on $S_{g+1}$, a surface of genus $g+1$. We will show that

$$
\mu\left(D\left(\psi_{g+1}\right)\right) \leq 1+2(g+1)=3+2 g .
$$

In other words we will show that $\psi_{g+1}$ has at most two more components than $\psi_{g}$.

Consider one of the handles of $S_{g+1}$, and let $L$ and $M$ be the longitude and meridian cycles in $\psi_{g+1}$ for this handle.

Choose an edge $e_{L}$ in $L$, but not in $M$, and then delete this edge. By Theorem 2.5

$$
\mu\left(D\left(\psi_{g+1}\right)\right) \leq \mu\left(D\left(\psi_{g+1} \backslash e_{L}\right)\right)+1 .
$$


Repeat this process for $M$ by choosing an edge $e_{M}$ in $M$, but not in $L$, to get

$$
\mu\left(D\left(\psi_{g+1} \backslash e_{L}\right)\right) \leq \mu\left(D\left(\psi_{g+1} \backslash\left\{e_{M}, e_{L}\right\}\right)\right)+1 .
$$

These two deletions yield a graph denoted $\psi_{g}$ which is no longer a pseudo-tree on $S_{g+1}$. It is, however, a pseudo-tree on the surface of genus $g$ obtained from $S_{g+1}$ by removing the handle under consideration. We now have

$$
\mu\left(D\left(\psi_{g+1}\right)\right) \leq \mu\left(D\left(\psi_{g}\right)\right)+2,
$$

as required.

Theorem 4.3. Let $G$ be a graph cellularly embedded on a surface of genus $g$. Then

$$
1 \leq \mu(D(G)) \leq f(G)+2 g .
$$

Proof. Let $\psi$ be a pseudo-tree of the graph $G$. Then $f(\psi)=1$, and by Theorem 4.2 we have $\mu(D(\psi)) \leq 1+2 g$, which means the theorem is true for $\psi$.

Now add edges to $\psi$, one by one, in order to obtain $G$. We obtain a sequence of graphs

$$
\psi=G_{0}, G_{1}, \ldots, G_{s-1}, G_{s}=G .
$$

The insertion of an edge increases the number of faces by exactly one, so for $i=0, \ldots, s-$ 1 we have

$$
f\left(G_{i+1}\right)=f\left(G_{i}\right)+1=f\left(G_{0}\right)+i+1 .
$$

By Theorem 2.5

$$
\begin{aligned}
\mu\left(D\left(G_{i+1}\right)\right) & \leq \mu\left(D\left(G_{i}\right)\right)+1 \\
& \leq \mu\left(D\left(G_{0}\right)\right)+i+1 .
\end{aligned}
$$

Since $\mu\left(D\left(G_{0}\right)\right) \leq f\left(G_{0}\right)+2 g$, we must have

$$
\begin{aligned}
\mu\left(D\left(G_{i+1}\right)\right) & \leq \mu\left(D\left(G_{0}\right)\right)+i+1 \\
& \leq f\left(G_{0}\right)+2 g+i+1 \\
& \leq f\left(G_{i+1}\right)+2 g .
\end{aligned}
$$

So $\mu\left(D\left(G_{i+1}\right)\right) \leq f\left(G_{i+1}\right)+2 g$ for each $i$, and hence the result. if

If $G$ is a graph cellularly embedded on a surface of genus $g$ then $G$ is called extremal

$$
\mu(D(G))=f(G)+2 g .
$$

Theorem 4.4. If $\psi$ is a spanning pseudo-tree of the extremal graph $G$, then $\psi$ is extremal.

Proof. Adding edges to $\psi$ one by one we obtain a sequence of graphs

$$
\psi=G_{0}, G_{1}, \ldots, G_{s}=G .
$$

In particular,

$$
G_{i-1}=G_{i} \backslash e
$$


where e is not a bridge. Suppose that $G_{i}$ is extremal. Then

$$
\mu\left(D\left(G_{i}\right)\right)=f\left(G_{i}\right)+2 g .
$$

Also,

$$
f\left(G_{i-1}\right)=f\left(G_{i}\right)-1 .
$$

By Theorem 2.5

$$
\begin{aligned}
\mu\left(D\left(G_{i-1}\right)\right) & \geq \mu\left(D\left(G_{i}\right)\right)-1 \\
& =f\left(G_{i}\right)+2 g-1 \\
& =f\left(G_{i-1}\right)+2 g .
\end{aligned}
$$

Now by Theorem 4.3

$$
\mu\left(D\left(G_{i-1}\right)\right)=f\left(G_{i-1}\right)+2 g,
$$

and so $G_{i-1}$ is extremal. Hence the result, by induction on $i$.

With two small modifications, Theorem 3.10 is also true for graphs cellularly embedded on surfaces of genus $g$ :

Theorem 4.5. Let $G$ be a graph cellularly embedded on a surface of genus $g$. Then the following statements are true.

(a) Let e be a bridge of $G$. Then $G / e$ is extremal if and only if $G$ is extremal.

(b) Let $v$ be a vertex of degree 2 with exactly one adjacent vertex, the two edges joining these vertices bounding a disc. Then $G \backslash v$ is extremal if and only if $G$ is extremal.

(c) Let $v$ be a vertex of degree 2 with two different adjacent vertices $x$ and $y$. Then $G /\{v, x\} /\{v, y\}$ is extremal if and only if $G$ is extremal.

(d) $G$ is extremal if and only if each block of $G$ is extremal.

(e) Let $G$ be extremal and e such that $G \backslash e$ is cellularly embedded. Then $G \backslash e$ is extremal.

Proof. This is exactly as in Theorem 3.10. In part (e) we note that each block of $G$ is a connected plane graph except for one, which must be cellularly embedded on the surface.

It follows that Lemma 3.11 is true for extremal graphs cellularly embedded on surfaces of genus $g$ :

Corollary 4.6. Let $G$ be an extremal graph cellularly embedded on a surface of genus $g$. Then each component of $D(G)$ only ever crosses itself on a bridge.

For any vertex $v \in V(G)$ we can define its degree $d(v)$ and we can also count the number of components of the link diagram of $G$ which pass close to $v$, denoting this by $\mu(v)$.

Theorem 4.7. Let $G$ be an extremal graph cellularly embedded on a surface of genus $g$, and let $v \in V(G)$, not a cut vertex. Then $d(v)=\mu(v)$. 


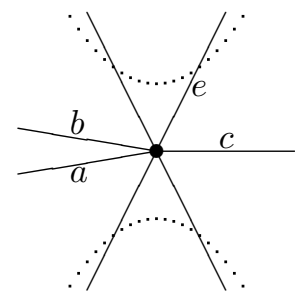

Figure 12: Two arcs, from the same component of the link diagram, passing close to $v$. There may be many other edges such as $a, b$, and $c$ incident with $v$, not shown here.

Proof. Suppose that $d(v)>\mu(v)$. Then there would be two arcs (from the same component of the link diagram) passing close to $v$, as in figure 12 . None of the edges incident with $v$ can be a bridge, or $v$ would be a cut vertex, so by part e of Theorem $4.5 G \backslash e$ is also torus extremal.

This process can be repeated until our two arcs, passing close to $v$, cross the same edge incident with $v$, as in figure 13. But this contradicts Corollary 4.6. Hence the result.

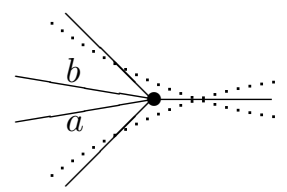

Figure 13: Two arcs, from the same component of the link diagram, passing close to $v$ and crossing the same edge incident with $v$. There may be many other edges such as $a$ and $b$ incident with $v$, not shown here.

\section{Concluding remarks}

It may also be possible to establish results like Theorem 3.2 and Corollary 3.4 for graphs cellularly embedded on surfaces of positive genus. They certainly appear to be true on the torus:

Conjecture 5.1. If $G$ is an extremal graph cellularly embedded on a torus then each face of $G$ is even.

Conjecture 5.2. If $G$ is an extremal graph cellularly embedded on a torus then $G$ is bipartite.

Next, let us make a few observations about plane graphs for which $\mu$ takes its smallest possible value. We leave the proofs of the results to the reader. Evidently, if $G$ is a tree then $\mu(D(G))=1$. Similarly, if $G$ is an odd cycle or its dual then $\mu(D(G))=1$.

Theorem 5.3. If $G$ is any cycle and $H=K_{3}^{*}$, then $\mu\left(D\left(G \oplus_{2} H\right)\right)=1$.

In the next two theorems, the two-sum can be taken at any edge of $G$, and in fact it can be replaced by the tensor product. 
Theorem 5.4. Let $G$ be a plane graph with $\mu(D(G))=1$ and $H$ be an even cycle or its dual. Then $\mu\left(D\left(G \oplus_{2} H\right)\right)=1$.

Theorem 5.5. Let $G$ be a tree and $H$ be any cycle. Then $\mu\left(D\left(G \oplus_{2} H\right)\right)=1$.

We finish by asking whether there are interesting families of graphs having specific values of $\mu$ greater than 1 but less than the maximum. Recall that the Petersen family $\mathcal{P}$ of graphs are those obtainable from $K_{6}$ by abstract $\mathrm{Y} \leftrightarrow \Delta$ moves. $\mathcal{P}$ has 7 members, including the Petersen graph itself, and it is of interest partly because of the following intriguing result [9]. (An intrinsically linked graph is one in which all spatial embeddings contain a non-splittable 2-component link.)

Theorem 5.6 (Robertson, Seymour, Thomas). $\mathcal{P}$ is the minor-minimal family for intrinsically linked graphs.

The graphs in $\mathcal{P}$ can all be cellularly embedded on the torus, but these embeddings are not unique. Suppose we focus on $K_{6}$, and suppose we restrict to embedded $\mathrm{Y} \leftrightarrow \Delta$ moves. Then, for any particular embedding of $K_{6}$ we will obtain a subfamily of $\mathcal{P}$ whose graphs all have the same value of $\mu$. Our preliminary results are indicated in the table below, where we have used the graph names given in [1]. ( $P_{10}$ is the Petersen graph.)

\begin{tabular}{c|c|c}
$\begin{array}{c}\text { the choice of } \\
\text { embedding }\end{array}$ & $\mu$ & $\begin{array}{c}\text { the family obtained using } \\
\text { embedded } \mathrm{Y} \leftrightarrow \Delta \text { moves }\end{array}$ \\
\hline (a) & 3 & $\mathcal{P}$ \\
(b) & 3 & $\mathcal{P} \backslash\left\{P_{10}, Q_{8}\right\}$ \\
(c) & 3 & $\mathcal{P} \backslash\left\{Q_{8}\right\}$ \\
(d) & 5 & $\mathcal{P} \backslash\left\{Q_{8}\right\}$ \\
(e) & 5 & $\mathcal{P} \backslash\left\{P_{10}, Q_{8}\right\}$ \\
(f) & 5 & $\mathcal{P} \backslash\left\{P_{10}\right\}$ \\
(g) & 7 & $\mathcal{P} \backslash\left\{P_{10}\right\}$
\end{tabular}

Similarly, the Heawood family $\mathcal{H}$ of graphs are those obtainable from $K_{7}$ by abstract $\mathrm{Y} \leftrightarrow \Delta$ moves. It has 20 members, which can all be cellularly embedded on the torus. It is shown in [1] that 14 of the graphs in $\mathcal{H}$ are intrinsically knotted. Again, choosing specific embeddings and restricting to embedded $\mathrm{Y} \leftrightarrow \Delta$ moves will yield subfamilies of graphs with constant $\mu$ values. 


\section{References}

[1] R. Hanaki, R. Nikkuni, K. Taniyama and A. Yamazaki, On intrinsically knotted or completely 3-linked graphs, Pacific Journal of Mathematics 252 (2011), 407-425.

[2] S. Huggett, On tangles and matroids, Journal of Knot Theory and its Ramifications 14 (2005), 919-929.

[3] X. Jin, F. Dong and E. Tay, On graphs determining links with maximal number of components via medial construction, Journal of Discrete Applied Mathematics 157 (2009), 3099-3110.

[4] M. Las Vergnas, On Eulerian partitions of graphs, Research Notes in Mathematics 34 (1979), 62-75.

[5] Y. Lin, S. D. Noble, X. Jin and W. Cheng, On plane graphs with link component number equal to the nullity, 2011, preprint.

[6] P. Martin, Remarkable Valuation of the Dichromatic Polynomial of Planar Multigraphs, Journal of Combinatorial Theory B 24 (1978), 318-324.

[7] E. G. Mphako, The component number of links from graphs, Proceedings of the Edinburgh Mathematical Society 45 (2002), 723-730.

[8] T. Pisanski, T. Tucker and A. Žitnik, Straight-ahead walks in Eulerian graphs, Discrete Mathematics 281 (2004), 237-246.

[9] N. Robertson, P. Seymour and R. Thomas, Sachs' linkless embedding conjecture, Journal of Combinatorial Theory Series B 64 (1995), 185-227. 